\section{CELIAC DISEASE IN TYPE 1 DIABETES CHILDREN FROM CONSTANTA COUNTY, ROMANIA}

C.M. Mihai ${ }^{1}$, A. Popp ${ }^{2}$, I. Durbala ${ }^{3}$, A. Balasa ${ }^{1}$, R.M. Stoicescu ${ }^{4}$, L. Mihai ${ }^{1}$

${ }^{1}$ Pediatric, Faculty of Medicine of Constanta, Constanta, ${ }^{2}$ Children's Health Institute, Bucharest, ${ }^{3}$ Immunology and Cellular Biology, Faculty

of Medicine of Constanta, ${ }^{4}$ Microbiology and Immunology, Faculty of Pharmacy of Constanta, Constanta, Romania

Introduction: Type 1 diabetes and celiac disease are both immunologic disorders where specific HLA alleles are associated with disease risk.

Objective: To estimate the prevalence of celiac disease (CD) and to describe the clinical features and HLA class II genotypes predictive of CD in our pediatric type 1 diabetes mellitus patients.

Material and methods: 65 patients underwent serologic screening with endomysial antibody and tissue transglutaminase antibody testing. CD was confirmed in serologic positive patients by intestinal biopsy.

All the patients also underwent HLA class II genotyping. Determination of HLA-DQ alleles was done by sequence-specific oligonucleotids hybridization after DNA amplification by polymerase chain reaction.

Results: A total of 65 patients with type 1 diabetes were tested, representing $12 \%$ of all type 1 diabetes patients from our county, and 4 had biopsy-proven $C D$, representing $6.1 \%$, for an estimated point prevalence of $7.0 \%$ (95\% Confidence Interval, $3.5 \%-12.1 \%$ ). Most CD-positive type 1 diabetes patients were asymptomatic and expressed an atrisk CD haplotype with at least one of but not both HLA DQ2 or DQ8. Patient 1: DRB1 3,3 (0301,0301)/ DQB1 2,2 (0201,0201). Patient 2: DRB1 4,13 (0401, 1302)/ DQB1 3,6 (0302, 0604). Patient 3: DRB1 3,16 (0301, 1601)/ DQB1 2, 5 (0201, 0502). Patient 4: DRB1 3,3 (0301, 0301)/ DQB1 2,2 (0201, 0201).

Conclusions: Celiac disease is not rare in our patients with type 1 diabetes, and most CD-positive patients with type 1 are asymptomatic irrespective of age at screening.

HLA-DQ genotype in children with T1DM may help in estimating the CD risk.

\section{USE OF MEDICINAL PLANTS FOR GESTATIONAL DIABETES IN BANGLADESH: A PRAGMATIC RANDOMIZED ETHNOPHARMACOLOGICAL SURVEY IN NARAIL DISTRICT}

\author{
M.A.H. Mollik ${ }^{1,2,3}$ \\ ${ }^{1}$ Epidemiology, Biostatistics, Community \\ Nutrition and Noncommunicable Diseases, \\ Peoples Integrated Alliance, ${ }^{2}$ Biotechnology and \\ Genetic Engineering, University of Development \\ Alternative, ${ }^{3}$ Health and Nutrition, Biogene Life \\ Care, Dhaka, Bangladesh
}

Gestational diabetes is a temporary condition that occurs during pregnancy. It affects about $12 \%$ of all pregnant women and increases the risk of developing diabetes for both mother and child. Gestational diabetes is prevalent in Bangladesh. The rural population mostly depends on traditional healers (THs) for treatment of this disease. The THs rely on decoctions made from medicinal plants or plant parts to treat gestational diabetes, which decoctions can vary widely between THs in the various districts of the country. The objective of this present study was to conduct an ethnopharmacological survey among the THs of Narail district, Bangladesh to learn about medicinal plants to treat gestational diabetes. In-depth information regarding medicinal plants type, preparation of medicines, ailments for which they are used, dosages, and side effects if any, were obtained from the THs. Medicinal plant samples were later identified at the Bangladesh National Herbarium. The collected information indicates that the following medicinal plants are used to treat gestational diabetes: Nigella sativa, Aconitum napellus, Agaricus albolutescens, Euphorbia cedrorum, Santalum album, Grewia asiatica, Ipomoea aquatica, Prunus communis, Plantago ovata, Phyllanthus emblica, Litsea liyuyingi, Ocimum tenuiflorum, Scoparia dulcis, Carica papaya, Bacopa monnieri, Tamarindus indica, Abrus precatorius, Dillenia indica, and Aloe vera. It was noted in this ethnopharmacological survey that the patients were quite satisfied with treatment by the THs. Since allopathic medicine does not have a cure for gestational diabetes, the above-mentioned medicinal plants are potentially interesting for discovery of novel compounds, which can be effective in controlling or curing gestational diabetes. 